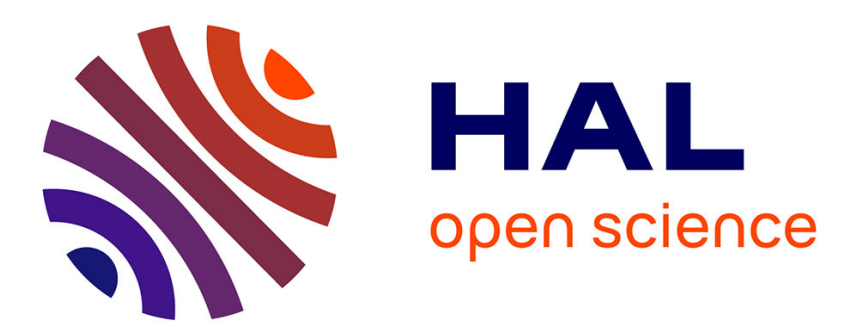

\title{
Degrees of Freedom of Ray-Based Models for mm-Wave Wideband MIMO-OFDM
}

\author{
Matthieu Roy, Stephane Paquelet, Matthieu Crussière
}

\section{To cite this version:}

Matthieu Roy, Stephane Paquelet, Matthieu Crussière. Degrees of Freedom of Ray-Based Models for mm-Wave Wideband MIMO-OFDM. 2019 IEEE Global Communications Conference (GLOBECOM), Dec 2019, Waikoloa, United States. 10.1109/GLOBECOM38437.2019.9013443 . hal-02305975

\section{HAL Id: hal-02305975 \\ https://hal.science/hal-02305975}

Submitted on 4 Oct 2019

HAL is a multi-disciplinary open access archive for the deposit and dissemination of scientific research documents, whether they are published or not. The documents may come from teaching and research institutions in France or abroad, or from public or private research centers.
L'archive ouverte pluridisciplinaire HAL, est destinée au dépôt et à la diffusion de documents scientifiques de niveau recherche, publiés ou non, émanant des établissements d'enseignement et de recherche français ou étrangers, des laboratoires publics ou privés. 


\title{
Degrees of Freedom of Ray-Based Models for mm-Wave Wideband MIMO-OFDM
}

\author{
Matthieu Roy ${ }^{\dagger}$, Stéphane Paquelet ${ }^{\dagger}$, Matthieu Crussière $^{\ddagger}$ \\ ${ }^{\dagger} \mathrm{b}<>$ com Rennes, France, firstname.lastname@b-com.com \\ $\ddagger$ Univ Rennes, INSA Rennes, IETR - UMR 6164 F-35000 Rennes, France
}

\begin{abstract}
In this paper we analyze the number of degrees of freedom needed to accurately capture and model wideband MIMO-OFDM channels. Two modeling approaches are considered, either examining each coefficient of the conventional inputoutput MIMO channel matrix or investigating the components constituting the physical ray-based propagation between the antenna arrays. As our analysis accounts for wideband channels, a time domain decomposition of MIMO channels into tapped delay lines is performed in each case. The efficiency of each approach is then evaluated in terms of number of taps to determine the most appropriate representation between either the conventional antenna-based one or the propagation-based one.

This analysis is performed analytically using the SalehValenzuela model which is recognized for its consistency for wideband as well as spatial modeling. We exploit the second-order statistics of the Channel Impulse Response to derive the taps weights.
\end{abstract}

Index Terms-ray-based model, MIMO, Saleh-Valenzuela

\section{INTRODUCTION}

Over the last decades, the envisioned size and complexity of Multiple-Input-Multiple-Output (MIMO) systems increased tremendously. From Single-Input-Single-Output (SISO) to small-scale MIMO systems, each MIMO link was modeled by an equivalent SISO (correlated or independent) link. As a consequence MIMO signal processing techniques were usually designed for SISO then mapped to MIMO channels [1]. However this approach becomes increasingly more complex as the antenna arrays get wider.

To tackle this issue, new scalable signal processing techniques for large-scale MIMO have emerged and constitute an highly active research topic today [2], [3]. Those advanced techniques take advantage of the clustered structure of the MIMO channel in the angular domain. Such feature is of particular importance in the millimeter wave band [2].

From a channel model point of view, most of the current stochastic modeling techniques are based on the framework of the well-known Saleh-Valenzuela channel model [4]. Originally introduced for SISO communications, it represents the propagation channel as a sum of a few clusters of decreasing average power with delay. The angular dimension of the propagation has been added later on for MIMO modeling in what is called the Extended Saleh-Valenzuela channel model [5].

From its introduction, the Saleh-Valenzuela model has been the focus of many studies in terms of verification through measurement campaigns and of characterization and calibration for various environments and frequencies [4], [5], [6], [7]. Despite its physical consistency, this model is considered as too complex for analytical computations and has essentially been used for simulation purposes. Only a few articles provide analytical results. Among which are the papers [8], [9], [10].
Contributions. In this paper we conduct an analytical analysis of the number of time domain coefficient (taps) required to model a wideband Saleh-Valenzuela MIMO-OFDM channel.

More precisely, we propose to compare two channel representations. For the first approach, each wideband link from a transmitting antenna to a receiving antenna is considered separately. This conventional approach is efficient for small-scale MIMO systems but scales poorly when increasing the antenna counts. As for the second approach, the clustered structure of the channel in the angular domain is exploited and we rather examine the frequency dependent cluster gains. This approach is expected to become more efficient in terms of number of taps for wide arrays as it no longer depends on the number of antennas.

We compare the two approaches using the Saleh-Valenzuela calibration measurements done in the $60 \mathrm{GHz}$ band by Lund University [6] and the IEEE 802.15.3c [7]. We show that only a few taps are sufficient to capture the channel behavior. The residual error is characterized herein by the model parameters through its first and second order statistics.

Notations. Upper case and lower case bold symbols are used for complex matrices and vectors of arbitrary size. $\langle.,$. denote the inner product between two vectors of $\mathbb{C}^{N} \cdot \vec{u}$ stands for a three-dimensional (3D) vector. $\vec{a} \cdot \vec{u}$ denote the inner product between two $3 \mathrm{D}$ vectors. $z^{*}$ denotes the conjugate of $z$. $[\mathbf{H}]_{p, q}$ is the element of matrix $\mathbf{H}$ at row $p$ and column $q$. $\|\mathbf{H}\|_{F}$ stands for the Frobenius norm. $\mathbf{H}^{H}$ and $\mathbf{H}^{T}$ denotes the conjugate transpose and the transpose matrices. $\mathbb{E}\{$.$\} and$ $\operatorname{Var}\{$.$\} denote the expectation and variance. \sharp$ denotes the cardinal of a set. Throughout this paper, the indexes $i, j, k, n$ denote iterators on receive antennas, transmit antennas, OFDM sub-carriers and time domain coefficients, respectively.

\section{MiMO Channel Model}

Let us consider the ray-based model widely used in the literature [2], [5], [11]. Following such approach, the MIMO channel is decomposed into several multipath components called clusters. Each cluster is defined by a frequency-dependent complex gain, a direction of departure and a direction of arrival.

\section{A. Multipath components}

The frequency gain of each cluster is described by a sum of $P$ intra-cluster rays

$$
c_{q}(f)=\sum_{p=1}^{P} \beta_{p, q} \mathrm{e}^{-2 \pi j f \tau_{p, q}}
$$

where $\tau_{p, q}$ is the arrival time of ray $p$ in cluster $q . \beta_{p, q}$ is the complex gain of ray $p$ of cluster $q$. The MIMO channel between 
the transmitter and the receiver is then given by the sum over all the clusters

$$
\begin{aligned}
\mathbf{H}(f)=\sqrt{N_{t} N_{r}} \sum_{q=1}^{Q} c_{q}(f) \mathrm{e}^{-2 \pi j f \tau_{q}} \\
\mathbf{e}_{\mathbf{r}}\left(\vec{u}_{r x, q}\right) \mathbf{e}_{\mathbf{t}}\left(\vec{u}_{t x, q}\right)^{H}
\end{aligned}
$$

where $Q$ denote the number of clusters, $\tau_{q}$ the arrival time of cluster $q$ relatively to the first cluster. $\vec{u}_{t x, p, q}\left(\vec{u}_{r x, p, q}\right)$ are 3D unit norm vectors denoting the direction of departure (arrival) of cluster $q$, also abbreviated DoD (DoA).

Considering the plane wave assumption, the functions $\mathbf{e}_{\mathbf{t}}(\vec{u})$ and $\mathbf{e}_{\mathbf{r}}(\vec{u})$ are called the steering vectors of the antenna array defined by

$$
\mathbf{e}_{\mathbf{t}}(\vec{u})=\frac{1}{\sqrt{N_{t}}}\left[\mathrm{e}^{2 j \pi \frac{\vec{a}_{t x, 1} \cdot \vec{u}}{\lambda}}, \cdots, \mathrm{e}^{2 j \pi \frac{\vec{a}_{t x, N_{t} \cdot \vec{u}}}{\lambda}}\right]^{T}
$$

where the antenna positions in the array are defined by the 3D vectors $\vec{a}_{t x, j}$ at the transmitter ( $\vec{a}_{r x, i}$ at the receiver respectively).

The Saleh-Valenzuela model relies on such ray-based model and specifies the distributions of the random variables $\tau_{q}, \tau_{p, q}$ and $\beta_{p, q}$ as detailed hereafter and illustrated in Fig. 1.

\section{B. Times of arrival}

The times of arrival of clusters follow a Poisson process of parameter $\Lambda$, called the cluster arrival rate. The first cluster arrives at $\tau_{1}=0$. The other times of arrival are then defined by the probability density function

$$
p\left(\tau_{q} \mid \tau_{q-1}\right)=\Lambda \mathrm{e}^{-\Lambda\left(\tau_{q}-\tau_{q-1}\right)}, \tau_{q}>\tau_{q-1}
$$

The times of arrival of intra-cluster rays also follow a Poisson process of parameter $\lambda$, called the ray arrival rate. The first ray arrives at $\tau_{1, q}=\tau_{q}$. Then the other times of arrival are defined by the following probability density function

$$
p\left(\tau_{p, q} \mid \tau_{p-1, q}\right)=\lambda \mathrm{e}^{-\lambda\left(\tau_{p, q}-\tau_{p-1, q}\right)}, \tau_{p, q}>\tau_{p-1, q} .
$$

\section{Complex gains}

Each ray is also characterized by a complex gain $\beta_{p, q}$ that follows a centered complex gaussian distribution. In this paper we normalize the average power of the very first ray to $\mathbb{E}\left\{\left|\beta_{1,1}\right|^{2}\right\}=1$. The variances of the coefficients follow a double exponential decay function parametrized by $\Gamma$ the cluster decay parameter and $\gamma$ the ray decay parameter

$$
\mathbb{E}\left\{\left|\beta_{p, q}\right|^{2}\right\}=\mathrm{e}^{-\tau_{q} / \Gamma} \mathrm{e}^{-\tau_{p, q} / \gamma} .
$$

\section{SECOND ORDER STATISTICS}

We first establish the expressions for the second order statistics of the channel, namely the frequency autocorrelation and the Power Spectral Density (PSD) as defined in [12], for both a single cluster and the full Saleh-Valenzuela channel. In this context the PSD matches the Power Delay Profile (PDP). Those expressions have already been derived in the context of UWB channel modeling in [10]. However our proofs (which are given in the appendices) neither require advanced point process theory or use Campbell's theorem.

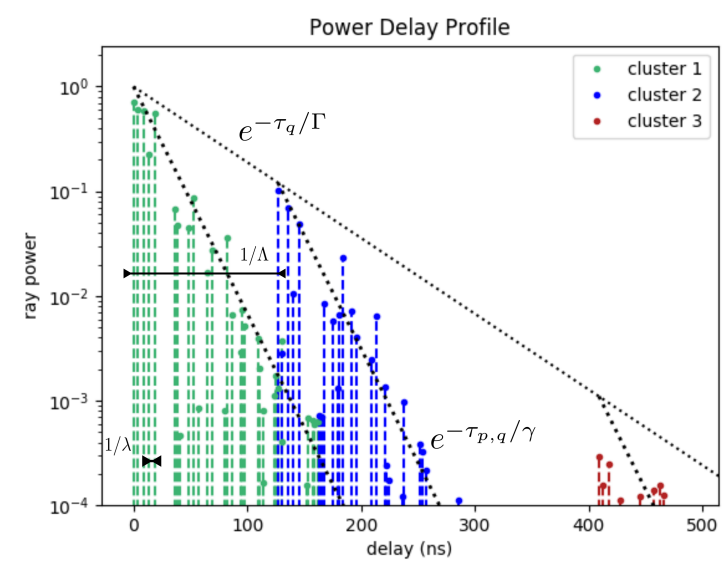

Fig. 1. A realization of a Saleh-Valenzuela channel.

\section{A. Single Cluster Analysis}

In this section we derive the expression of the autocorrelation and PDP of a single cluster of the Saleh-Valenzuela channel model. The complex gain of a cluster is given by

$$
c_{q}(f)=\sum_{p=1}^{P} \beta_{p, q} \mathrm{e}^{-2 \pi j f \tau_{p, q}} .
$$

Due to the exponentially decaying intra-cluster powers with increasing delay, only a finite number of dominant rays are distinguishable. $P$ is chosen high enough to embrace the whole multi-path power while keeping computational complexity low for simulation purposes. For analytical computation convenience, we will consider that $P \rightarrow \infty$. The details of the derivations are given in appendix A-A. We obtain the formula

$$
R_{c_{q}}(\Delta f)=\mathbb{E}\left\{\left|\beta_{1, q}\right|^{2}\right\}\left(1+\frac{\lambda \gamma}{1-2 \pi j \Delta f \gamma}\right) .
$$

It is noticeable that this expression consists of two terms, the former being constant and the latter vanishing as the frequency difference increases. This feature is also observed in [8]. We obtain the PDP by applying the Fourier Transform on the autocorrelation,

$$
S_{c_{q}}(\tau)=\mathbb{E}\left\{\left|\beta_{1, q}\right|^{2}\right\}\left(\delta(\tau)+\lambda \mathrm{e}^{-\tau / \gamma} u(\tau)\right)
$$

where $u(\tau)$ is the Heaviside step function. The average power of the cluster is

$$
P_{c_{q}}=\mathbb{E}\left\{\left|c_{q}\right|^{2}\right\}=\mathbb{E}\left\{\left|\beta_{1, q}\right|^{2}\right\}(1+\lambda \gamma) .
$$

Using a recurrence we compute $\mathbb{E}\left\{\left|\beta_{1, q}\right|^{2}\right\}$

$$
\mathbb{E}\left\{\left|\beta_{1, q}\right|^{2}\right\}=\mathbb{E}\left\{\left|\beta_{1, q-1}\right|^{2}\right\}\left(\frac{1}{1+\frac{1}{\Lambda \Gamma}}\right)=\left(\frac{1}{1+\frac{1}{\Lambda \Gamma}}\right)^{q-1}
$$

\section{B. Saleh-Valenzuela Analysis}

In this section we derive the expression of the autocorrelation and PDP of the full Saleh-Valenzuela. The complex frequency gain of the channel is given by

$$
H(f)=\sum_{q=1}^{Q} \sum_{p=1}^{P} \beta_{p, q} \mathrm{e}^{-2 \pi j f\left(\tau_{p, q}+\tau_{q}\right)} .
$$


As previously done, we will consider that $P \rightarrow \infty$ and $Q \rightarrow \infty$. The detail of the calculus of the autocorrelation function is given in appendix A-B. In particular we find that the random process $H$ is WSS. This yields the expression

$$
R_{H}(\Delta f)=\left(1+\frac{\Lambda \Gamma}{1-2 \pi j \Delta f \Gamma}\right)\left(1+\frac{\lambda \gamma}{1-2 \pi j \Delta f \gamma}\right) .
$$

This expression can be expressed as the product of the autocorrelations of two single-exponentially decaying channels. Then we apply the Fourier Transform to the autocorrelation function to get the PDP

$$
\begin{aligned}
S_{H}(\tau) & =\delta(\tau)+\Lambda \mathrm{e}^{-\tau / \Gamma} u(\tau)+\lambda \mathrm{e}^{-\tau / \gamma} u(\tau) \\
& +\Lambda \lambda \frac{\Gamma \gamma}{\Gamma-\gamma}\left(\mathrm{e}^{-\tau / \gamma}-\mathrm{e}^{-\tau / \Gamma}\right) u(\tau)
\end{aligned}
$$

where $u(\tau)$ is the Heaviside step function.

The average power of the Saleh-Valenzuela channel is then given by

$$
P_{H}=\mathbb{E}\left\{|H|^{2}\right\}=(1+\lambda \gamma)(1+\Lambda \Gamma) .
$$

\section{MOdELING SISO CHANNELS}

In this section we consider a SISO-OFDM setup that operates on a bandwidth $\mathcal{B}=[-B / 2, B / 2]$, divided into $N_{f}$ subcarriers spaced by $\Delta B=B / N_{f}$. The frequency-domain channel gain at subcarrier $k$ is denoted $H[k]$. The model complexity is measured in terms of the number of time domain coefficients. To do so we perform an IFFT on the frequency domain channel gain. The $N_{f}$ taps are given by

$$
h_{n}=\frac{1}{N_{f}} \sum_{k=0}^{N_{f}-1} H[k] \mathrm{e}^{2 \pi j n k / N_{f}}, n \in \llbracket 0, N_{f}-1 \rrbracket .
$$

Although the sum (10) can be very large, the channel power is in practice concentrated on a small set of time domain coefficients $\mathcal{N}\left(\sharp \mathcal{N} \ll N_{f}\right)$ so most of them are not significant and can be discarded. As a consequence we will only keep a small set of coefficients $\mathcal{N}$

$$
H_{\mathcal{N}}[k]=\sum_{n \in \mathcal{N}} h_{n} \mathrm{e}^{-2 \pi j n k / N_{f}} .
$$

This complexity reduction introduces an error that is quantified herein by the Mean Square Error (MSE) criterion. This operation is typically performed in OFDM channel estimation for noise reduction. The resulting systematic error is then called the bias. Using the orthogonality between the complex exponential functions, the MSE induced by this simplification is given by

$$
\mathbb{E}\left\{\left\|\tilde{H}_{\mathcal{N}}-H\right\|^{2}\right\}=N_{f} \sum_{n \notin \mathcal{N}} \mathbb{E}\left\{\left|h_{n}\right|^{2}\right\}=N_{f} \sum_{n \notin \mathcal{N}} \operatorname{Var}\left(h_{n}\right) .
$$

The variances can be computed by solving the integral

$$
\operatorname{Var}\left(h_{n}\right)=\int_{-\infty}^{\infty} S_{H}(\tau) D_{N_{f}}^{2}\left(\pi \frac{n-\tau B}{N_{f}}\right) \mathrm{d} \tau
$$

where $D_{N}(x)$ denotes the normalized Dirichlet kernel

$$
D_{N}(x)=\frac{\sin (N x)}{N \sin (x)} .
$$
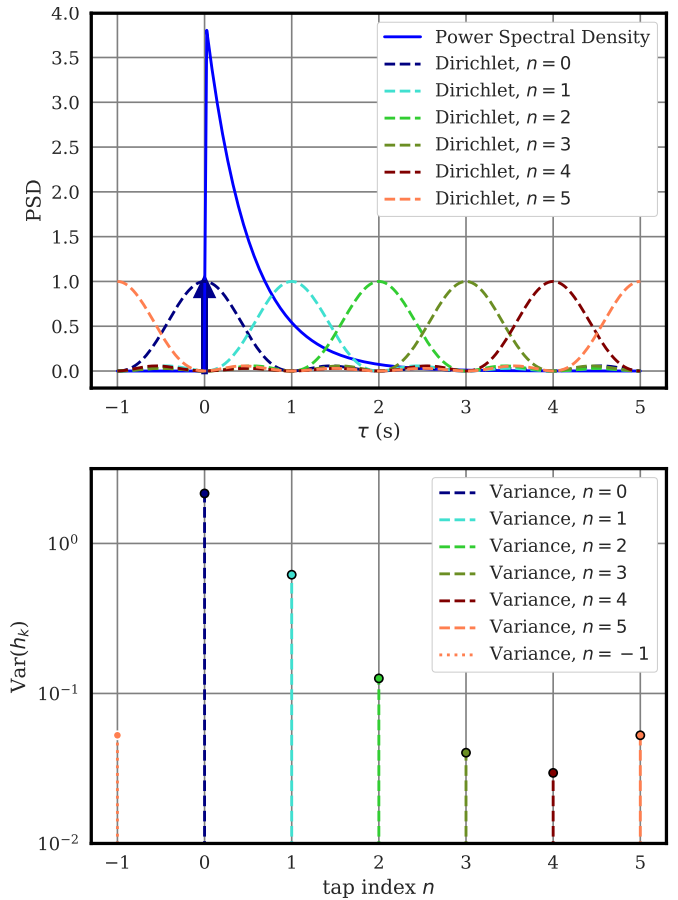

Fig. 2. Relation between the PDP of a channel and the variance of the time domain taps. Each tap is obtained by the convolution of the corresponding Dirichlet kernel with the PSD of the channel.

This expression of the variance provides a simple way to compute the error induced by a particular truncation of the time domain coefficients. Variance formula (12) is illustrated on Fig. 2. The leftmost bin denoted $n=-1$ is a replicate of the last bin $n=5$ as the Dirichlet kernel is a periodic function.

\section{Modeling MiMO CHANnEls}

We consider two modeling approaches. First we analyze each frequency-dependent MIMO channel matrix coefficient. Each one of those coefficients represents the equivalent SISO channel from an emitting antenna to a receiving antenna. We called this method antenna based modeling.

On one other hand, we consider the channel as a sum of several multipath components also called clusters. We can take advantage of this feature and analyze the channel through those frequency-dependent clusters. We called this method propagation based modeling. Both approaches are illustrated by Fig. 3 and Fig. 4.

In this section, $\mathbf{H}$ is a 3-dimensional tensor whose elements are denoted $H_{i, j}[k]$. The approximated channel matrix is denoted $\mathbf{H}_{\mathcal{N}}$. The error is evaluated with the MSE criterion,

$$
\mathbb{E}\left\{\left\|\mathbf{H}_{\mathcal{N}}-\mathbf{H}\right\|^{2}\right\}=\mathbb{E}\left\{\sum_{i, j, k}\left|H_{\mathcal{N} i, j}[k]-H_{i, j}[k]\right|^{2}\right\} .
$$

\section{A. Antenna based modeling}

The channel gain from antenna $j$ to antenna $i$ on subcarrier $k$ is given by $H_{i, j}[k]$. Each one of the $N_{r} \times N_{t}$ channels can be decomposed into time domain coefficients as in 


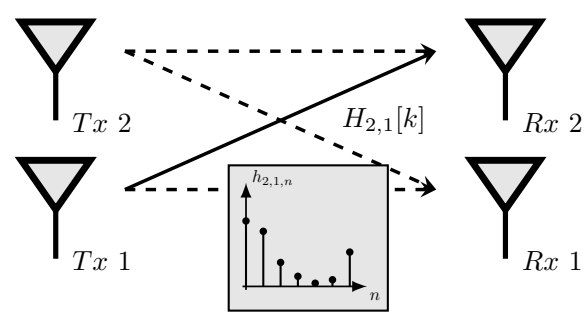

Fig. 3. Antenna based modeling.

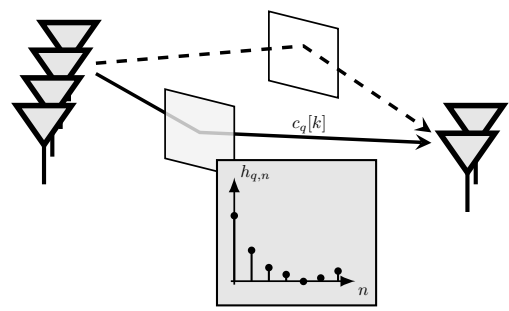

Fig. 4. Propagation based modeling

(10). The resulting $h_{i, j, n}$ are obviously correlated over the different antennas. We can then apply the methodology of IV. Considering that only the subset $\mathcal{N}$ of coefficient is not discarded on each SISO-equivalent link, the MSE is given by

$$
\mathbb{E}\left\{\left\|\tilde{\mathbf{H}}_{\mathcal{N}}-\mathbf{H}\right\|^{2}\right\}=N_{r} N_{t} N_{f} \sum_{n \notin \mathcal{N}} \operatorname{Var}\left(h_{n}\right) .
$$

One can notice that the results doesn't depend on the correlations between the time domain coefficients.

\section{B. Propagation based modeling}

The gain of each cluster at sub-carrier $k$ can be obtained by sampling of $c_{q}[k]$ (1). Then we follow the same methodology as in IV. This cluster gain can be represented in the time domain

$$
h_{q, n}=\frac{1}{N_{f}} \sum_{k=0}^{N_{f}-1} c_{q}[k] \mathrm{e}^{2 \pi j n k / N_{f}} .
$$

Cluster $q$ can then be represented by a set of $N_{f}$ time domain coefficients $h_{q, n}$, most of those being not significant and can be discarded. For each cluster $q$ we keep a set of coefficients $\mathcal{N}_{q}$. The MSE introduced by this truncation is given by

$$
\mathbb{E}\left\{\left\|\tilde{\mathbf{H}}_{\mathcal{N}}-\mathbf{H}\right\|^{2}\right\}=N_{r} N_{t} \sum_{q=1}^{Q} \mathbb{E}\left\{\left\|c_{q, \mathcal{N}_{q}}-c_{q}\right\|^{2}\right\}
$$

where the right hand side is given by the uniform independent phases of the cluster gains. The terms of the sum are given by

$$
\mathbb{E}\left\{\left\|\tilde{c}_{q, \mathcal{N}}-c_{q}\right\|^{2}\right\}=N_{f} \sum_{k \notin \mathcal{N}_{q}} \mathbb{E}\left\{\left|h_{q, n}\right|^{2}\right\}=N_{f} \sum_{n \notin \mathcal{N}_{q}} \operatorname{Var}\left(h_{q, n}\right) .
$$

It is expressed as a sum of variances, whose expression is :

$$
\operatorname{Var}\left(h_{q, n}\right)=\int_{-\infty}^{\infty} S_{c_{q}}(\tau) D_{N_{f}}^{2}\left(\pi \frac{n-\tau B}{N_{f}}\right) \mathrm{d} \tau .
$$

TABLE I

SALEH-VALENZUELA PARAMETERS

\begin{tabular}{c|cccc}
\hline & $\Lambda(1 / n s)$ & $\Gamma(n s)$ & $\lambda(1 / n s)$ & $\gamma(n s)$ \\
\hline [6] Lund Uni. & 0.2 & 8.7 & 1.1 & 4.7 \\
[7] Office & 0.028 & 134 & 0.760 & 59 \\
[7] Library & 0.25 & 12 & 4 & 7
\end{tabular}

Based on the expression of $S_{c_{q}}$ in (16), equation (15) can be further simplified using (7)

$$
\mathbb{E}\left\{\left\|\tilde{c}_{q, \mathcal{N}}-c_{q}\right\|^{2}\right\}=N_{f}\left(\frac{1}{1+\frac{1}{\Lambda \Gamma}}\right)^{q-1} \sum_{n \notin \mathcal{N}_{q}} \operatorname{Var}\left(h_{1, n}\right) .
$$

Once the variances are evaluated, the error induced by the choices of $\mathcal{N}_{q}$ can be easily calculated.

\section{RESUlts}

Parameters $\Lambda, \Gamma, \lambda, \gamma$ characterizing the Saleh-Valenzuela have been measured in a wide variety of situations in both $\mathrm{GHz}$ as well as millimeter waves frequency bands. We used the $60 \mathrm{GHz}$ parameters from Lund University [6] and the IEEE 802.15.3c office and library setups [7] which are summarized in Table I. In the previous sections we saw that the total channel power depends on $\Lambda, \Gamma, \lambda, \gamma$. To come by with comparable results we will consider the relative error

$$
\frac{\mathbb{E}\left\{\left\|\tilde{\mathbf{H}}_{\mathcal{N}}-\mathbf{H}\right\|^{2}\right\}}{\mathbb{E}\left\{\|\mathbf{H}\|^{2}\right\}}=\frac{\mathbb{E}\left\{\left\|\tilde{\mathbf{H}}_{\mathcal{N}}-\mathbf{H}\right\|^{2}\right\}}{N_{r} N_{t} N_{f}(1+\Lambda \Gamma)(1+\lambda \gamma)} .
$$

We performed numerical evaluations of the relative error for a typical LTE frame structure (also valid for 5G NR frames) of $10 \mathrm{MHz}$ total bandwidth (In fact the occupied bandwidth is $B=9 \mathrm{MHz}$ due to edge effects), an inter-carrier spacing of $\Delta f=15 \mathrm{kHz}$ and $N_{f}=600$ sub-carriers. We evaluate the error for both antenna based modeling and propagation based modeling. The MSE of the channel can then be derived using (11) and (15). The results are given in Fig. 5. The antenna based approach (dashed lines) yields a smaller MSE for small arrays while the propagation based approach (red solid line) is preferable for large arrays.

Those MSE formulas can be inverted using iterative techniques to assess the number of taps required to achieve a desired MSE. This yields Fig. 6 where the boundary between both regimes is even further evidenced. The antenna based approach is as expected more efficient for small number of antennas whereas the propagation based approach is more efficient for large-scale MIMO. The transition from the former to the later approaches happens surprisingly fast at $N_{r} \times N_{t}>10$ at most. This configuration is easily attained if we consider multiple receive antennas.

We neglected the cluster angular spread. Indeed for mm-waves setups where $N_{r} \times N_{t}<10$ this hypothesis is reasonable. Wider arrays could be considered by splitting the clusters into multiple subclusters depending on $N_{r} \times N_{t}$. The flat curves of Fig. 6 would be slightly increasing and the previous observations and conclusions wouldn't change. 

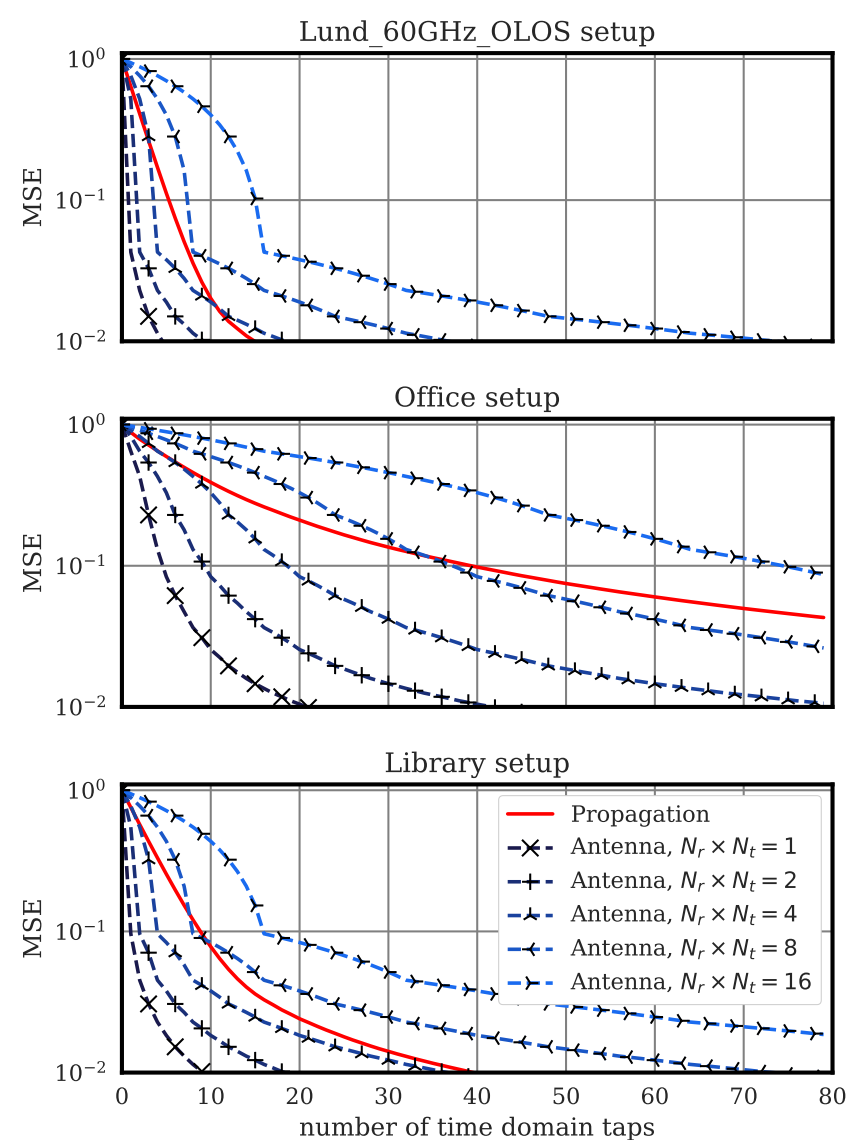

Fig. 5. MSE as a function of the number of taps. The antenna based approach is represented by the dashed lines and the propagation based approach is represented by the red solid line.

\section{CONCLUSION}

In this paper the number of time domain coefficients required to model a MIMO-OFDM Saleh-Valenzuela channel for a given error has been analyzed. This evaluation is based on the secondorder channel statistics which enabled the comparison between two representation methods. The matrix coefficient (resp. multipath) based approach is suitable for small-scale (resp. large-scale) MIMO. We performed extensive numerical evaluations using calibrated Saleh-Valenzuela models available in the literature and found out that the transition between those two representations arises at surprisingly small number of antennas. To highlight this property, we made the assumption that array angular resolution is limited resulting in unresolvable intra-clusters DoDs and DoAs.

In further studies this assumption could be removed by splitting clusters into multiple sub-clusters with parametrization scaled accordingly.

The channel model bias, induced by the discarded taps and derived in this study, can be further exploited for channel estimation error evaluation.

\section{APPENDIX A}

\section{STATISTICAL AUTOCORRELATION FUNCTIONS}

\section{A. Cluster channel gain}

In (4) the phases of the complex gains $\beta_{p, q}$ are all independent resulting in $\mathbb{E}\left\{\beta_{p_{1}, q} \beta_{p_{2}, q}^{*}\right\}=\delta_{p_{1}, p_{2}} \mathbb{E}\left\{\left|\beta_{p_{1}, q} \beta_{p_{2}, q}\right|\right\}$. The
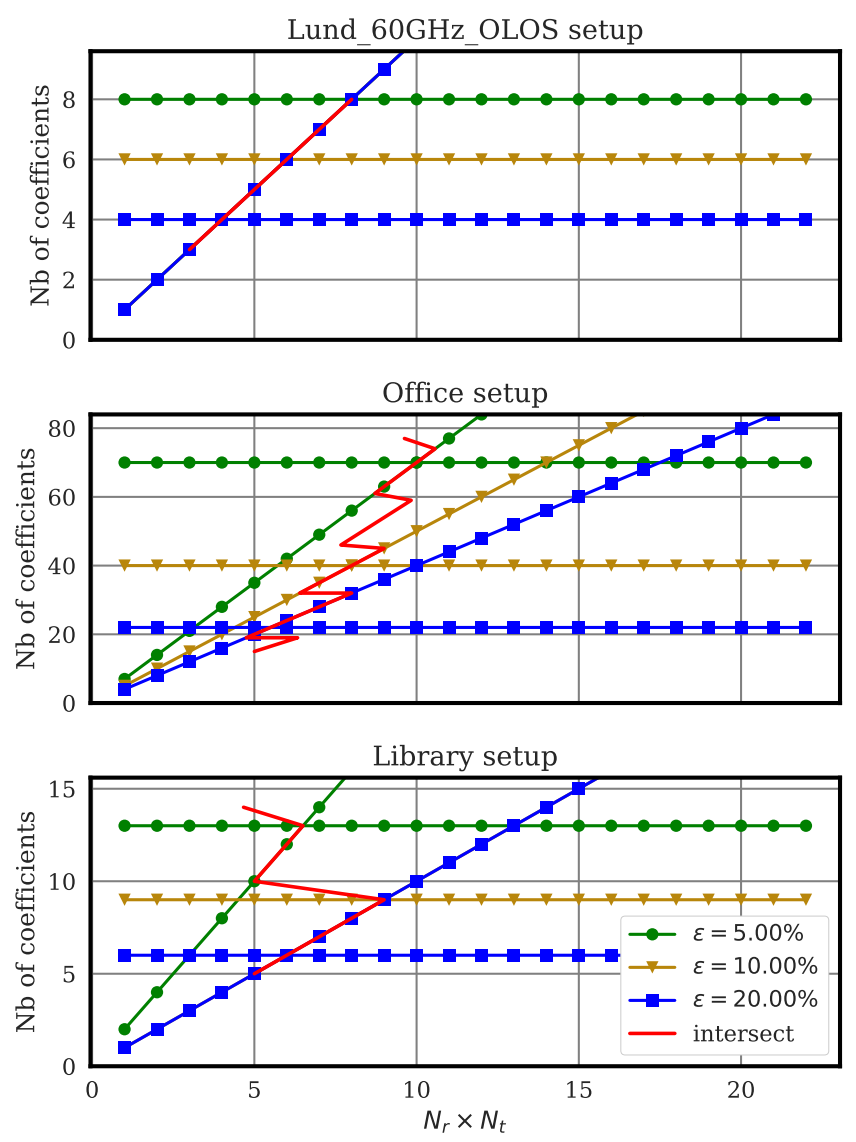

Fig. 6. Number of taps required to achieve a desired MSE. After $N_{r} \times N_{t}>10$, the propagation based approach is always more efficient. The boundary between both regimes for any MSE is given by the red solid curve.

autocorrelation is then given by

$$
R_{c_{q}}\left(f, f^{\prime}\right)=\sum_{p=1}^{\infty} \mathbb{E}\left\{\left|\beta_{p, q}\right|^{2} \mathrm{e}^{-2 \pi j\left(f-f^{\prime}\right) \tau_{p, q}}\right\} .
$$

The random process is Wide Sense Stationary (WSS). We set $\Delta f=f^{\prime}-f$. Then using conditional expectation on $\tau_{p, q}$ and the exponential decay $\mathbb{E}\left\{\left|\beta_{p, q}\right|^{2} \mid \tau_{p, q}\right\}=\mathbb{E}\left\{\left|\beta_{1, q}\right|^{2}\right\} \mathrm{e}^{-\tau_{p, q} / \gamma}$ we get

$$
R_{c_{q}}(\Delta f)=\mathbb{E}\left\{\left|\beta_{1, q}\right|^{2}\right\} \sum_{p=1}^{\infty} \mathbb{E}_{\tau_{p, q}}\left\{\mathrm{e}^{(2 \pi j \Delta f-1 / \gamma) \tau_{p, q}}\right\} .
$$

Then using a recurrence over $p$ we can prove that

$$
\mathbb{E}_{\tau_{p, q}}\left\{\mathrm{e}^{(2 \pi j \Delta f-1 / \gamma) \tau_{p, q}}\right\}=\lambda^{p-1} \frac{1}{\left(\frac{1}{\gamma}+\lambda-2 \pi j \Delta f\right)^{p-1}} .
$$

Equation (17) is a geometric series whose ratio $r=\frac{1}{\frac{1}{\lambda \gamma}+1-2 \pi j \Delta f / \lambda}$ always verify $|r|<1$ so that the series converges to

$$
R_{c c}(\Delta f)=\mathbb{E}\left\{\left|\beta_{1, q}\right|^{2}\right\}\left(1+\frac{\lambda \gamma}{1-2 \pi j \gamma \Delta f}\right) .
$$




\section{B. Saleh-Valenzuela channel gain}

Still using the independence of the phases of the complex gains in (8), we get a simplified expression. We find that this channel is also WSS. We obtain the expression

$$
R_{H}(\Delta f)=\sum_{q=1}^{\infty} \sum_{p=1}^{\infty} \mathbb{E}\left\{\left|\beta_{p, q}\right|^{2} \mathrm{e}^{2 \pi j \Delta f\left(\tau_{p, q}+\tau_{q}\right)}\right\} .
$$

Using a conditional expectation on the $\tau_{p, q}$ and the $\tau_{q}$ and using the double exponential decay formula of the Saleh-Valenzuela channel $\mathbb{E}\left\{\left|\beta_{p, q}\right|^{2} \mid \tau_{p, q}\right\}=\mathrm{e}^{-\tau_{q} / \Gamma} \mathrm{e}^{-\tau_{p, q} / \gamma}$ , we get an expression similar to (17)

$$
R_{H}(\Delta f)=\sum_{q=1}^{\infty} \sum_{p=1}^{\infty} \mathbb{E}\left\{\mathrm{e}^{-(1 / \Gamma-2 \pi j \Delta f) \tau_{q}} \mathrm{e}^{-(1 / \gamma-2 \pi j \Delta f) \tau_{p, q}}\right\}
$$

Because the random variables $\tau_{q}$ and $\tau_{p, q}$ are independent, we can split the expectation. Using the result of (A-A) on both parts, the equation can be rewritten as the product of two geometric series yielding the result (9).

\section{APPENDIX B}

\section{VARIANCES OF TIME DOMAIN COEFFICIENTS}

The variance of the time domain coefficient $h_{n}$ is

$\mathbb{E}\left\{h_{n} h_{n}^{*}\right\}=\frac{1}{N_{f}^{2}} \sum_{k_{1}=0}^{N_{f}-1} \sum_{k_{2}=0}^{N_{f}-1} \mathbb{E}\left\{H\left[k_{1}\right] H\left[k_{2}\right]^{*}\right\} \mathrm{e}^{-2 \pi j\left(k_{2}-k_{1}\right) n / N_{f}}$.

Where we can recognize the autocorrelation $\mathbb{E}\left\{H\left[k_{1}\right] H\left[k_{2}\right]^{*}\right\}=R_{H, H}\left[k_{2}-k_{1}\right]$. We use the autocorrelation expression from (18) with $\Delta f=\left(k_{2}-k_{1}\right) \Delta B$

$$
\begin{aligned}
\mathbb{E}\left\{H\left[k_{1}\right] H\left[k_{2}\right]^{*}\right\}= & \sum_{q=1}^{\infty} \sum_{p=1}^{\infty} \mathbb{E}\left\{\left|\beta_{p, q}\right|^{2}\right. \\
& \left.\mathrm{e}^{2 \pi j\left(k_{2}-k_{1}\right) \Delta B\left(\tau_{p, q}+\tau_{q}\right)}\right\} .
\end{aligned}
$$

We insert this expression into the variance formula and get

$$
\begin{aligned}
\mathbb{E}\left\{h_{n} h_{n}^{*}\right\}= & \frac{1}{N_{f}^{2}} \sum_{q=1}^{\infty} \sum_{p=1}^{\infty} \mathbb{E}\left\{\left|\beta_{p, q}\right|^{2}\right. \\
& \left.\sum_{k_{1}=0}^{N_{f}-1} \sum_{k_{2}=0}^{N_{f}-1} \mathrm{e}^{2 \pi j\left(k_{2}-k_{1}\right) \Delta B\left(\tau_{p, q}+\tau_{q}-n /\left(N_{f} \Delta B\right)\right)}\right\}
\end{aligned}
$$

where the sums equals $D_{N_{f}}^{2}\left(\pi \Delta B\left(\tau_{p, q}+\tau_{q}-n / B\right)\right)$ resulting in

$$
\begin{aligned}
\mathbb{E}\left\{h_{n} h_{n}^{*}\right\}= & \sum_{q=1}^{\infty} \sum_{p=1}^{\infty} \mathbb{E}\left\{\left|\beta_{p, q}\right|^{2}\right. \\
& \left.D_{N_{f}}^{2}\left(\pi \Delta B\left(\tau_{p, q}+\tau_{q}-n / B\right)\right)\right\} .
\end{aligned}
$$

We introduce the continuous function defined for $x \in \mathbb{R}$

$$
f(x)=\sum_{q=1}^{\infty} \sum_{p=1}^{\infty} \mathbb{E}\left\{\left|\beta_{p, q}\right|^{2} D_{N_{f}}^{2}\left(\pi \Delta B\left(\tau_{p, q}+\tau_{q}-x / B\right)\right)\right\} .
$$

After a first Inverse Fourier Transform and substituting $y=B \tau_{p, q}+B \tau_{q}-x$

$$
\begin{aligned}
\sum_{q=1}^{\infty} \sum_{p=1}^{\infty} \mathbb{E}\left\{\left|\beta_{p, q}\right|^{2} \mathrm{e}^{2 \pi j \nu\left(\tau_{p, q}+\tau_{q}\right)}\right\} \\
\int_{-\infty}^{\infty} D_{N_{f}}^{2}\left(\pi y / N_{f}\right) \mathrm{e}^{-2 \pi j \nu y / B} \mathrm{~d} y .
\end{aligned}
$$

The first line matches the autocorrelation function of the channel $R(\nu)$. The second part is the Fourier Transform of the squared Dirichlet kernel. We retrieve the function $f$ using a FT:

$$
f(x)=\int_{-\infty}^{\infty} S_{H}(\tau) D_{N_{f}}^{2}\left(\pi(\tau B-x) / N_{f}\right) \mathrm{d} \tau .
$$

Applying this formula for $x=n$, we get the expression.

\section{REFERENCES}

[1] G. Stuber, J. Barry, S. McLaughlin, Ye Li, M. Ingram, and T. Pratt, "Broadband MIMO-OFDM wireless communications," Proceedings of the IEEE, vol. 92, no. 2, pp. 271-294, Feb. 2004

[2] L. L. Magoarou and S. Paquelet, "Parametric channel estimation for massive MIMO," arXiv preprint arXiv:1710.08214, 2017.

[3] A. Khlifi and R. Bouallegue, "Hybrid LS-LMMSE Channel Estimation Technique for LTE Downlink Systems," International Journal of Next-Generation Networks, vol. 3, no. 4, pp. 1-13, Dec. 2011

[4] A. A. M. Saleh and R. Valenzuela, "A statistical model for indoor multipath propagation," IEEE Journal on selected areas in communications, vol. 5, no. 2, pp. 128-137, 1987.

[5] Q. Spencer, B. Jeffs, M. Jensen, and A. Swindlehurst, "Modeling the statistical time and angle of arrival characteristics of an indoor multipath channel," IEEE Journal on Selected Areas in Communications, vol. 18, no. 3, pp. 347-360, Mar. 2000.

[6] C. Gustafson, K. Haneda, S. Wyne, and F. Tufvesson, "On mm-Wave Multipath Clustering and Channel Modeling," IEEE Transactions on Antennas and Propagation, vol. 62, no. 3, pp. 1445-1455, Mar. 2014.

[7] S.-K. Yong et al., "G3c channel modeling sub-commitee final report," Mar. 2007.

[8] M. S. Derpich and R. Feick, "Second-Order Spectral Statistics for the Power Gain of Wideband Wireless Channels," IEEE Transactions on Vehicular Technology, vol. 63, no. 3, pp. 1013-1031, Mar. 2014.

[9] _ - "On the second order power spectral statistics of wideband indoor microwave channels," in 21st Annual IEEE International Symposium on Personal, Indoor and Mobile Radio Communications. Istanbul, Turkey: IEEE, Sep. 2010, pp. 335-340.

[10] A. Meijerink and A. F. Molisch, "On the Physical Interpretation of the SalehValenzuela Model and the Definition of Its Power Delay Profiles," IEEE Transactions on Antennas and Propagation, vol. 62, no. 9, pp. 4780-4793, Sep. 2014.

[11] C. Liu, E. Skafidas, and R. Evans, "Angle of arrival extended S-V model for the $60 \mathrm{GHz}$ wireless indoor channel," in 2007 Australasian Telecommunication Networks and Applications Conference. Christchurch, New Zealand: IEEE, Dec. 2007, pp. 181-185.

[12] B. Hajek, Random Processes for Engineers, 1st ed. Cambridge University Press, Mar. 2015. 